\title{
Quasi-static modelling of compliant mechanisms: application to a 2-DOF underactuated finger
}

\author{
C. Quennouelle and C. Gosselin \\ Laboratoire de robotique, Département de génie mécanique, Université Laval, Québec, QC, Canada
}

Received: 23 February 2010 - Revised: 30 June 2010 - Accepted: 19 August 2010 - Published: 8 February 2011

\begin{abstract}
In this paper, the kinematostatic and the quasi-static models of parallel mechanisms are applied to underactuated mechanisms. Both models are extended to the cases for which the actuated joints are not kinematically independent, and for which the external loads are function of the configuration of the mechanism, the grasped object being considered as not perfectly rigid. An application to a 2-DOF underactuated compliant finger is then presented with details about the implementation of the kinematostatic and the quasi-static models. Finally, some numerical results are given that illustrate possible contributions of these models for the analysis and the control of underactuated mechanisms.
\end{abstract}

This paper was presented at the IFToMM/ASME International Workshop on

Underactuated Grasping (UG2010), 19 August 2010, Montréal, Canada.

\section{Introduction}

Compliant mechanisms, i.e., mechanisms built with elastic joints, offer several advantages compared to conventional mechanisms: reduction of wear, clearance and backlash, compactness, no need for lubrication, simplified assembly, etc. However, due to their particular behaviour - namely the coupling between applied forces/torques and motion, and the parasitic deformations (a single joint can have several degrees of freedom (Su, 2009), the classical kinematic models are not sufficient to accurately describe them. Therefore, some models taking into account their specific behaviour have recently been developed. These models describe the configuration of the mechanisms (represented by a minimal set of kinematic parameters, i.e., the generalized coordinates) as a function of some external static parameters, such as the external loads and the commanded positions of the actuators (Sun et al., 1997; Carricato, 1998; Quennouelle and Gosselin, 2009). Due to the multiple degrees of freedom that can be required for an accurate modelling of a single compliant joint, any compliant mechanism can be considered as an underactuated mechanism, i.e., with fewer actuators than degrees of mobility. Therefore, the kinematostatic model (KSM) and

Correspondence to: C. Quennouelle (c.quennouelle@gmail.com) the quasi-static model (QSM) of compliant mechanisms appear to be useful tools for the study of underactuated mechanisms (UMs) in general.

On the other hand, in the recent years, research initiatives on underactuated robotic hands led to a miniaturization of these systems and several interesting compliant prototypes have been proposed (Lotti et al., 2005; Boudreault and Gosselin, 2006; Doria and Birglen, 2009). For example, Fig. 1 shows a compliant gripper designed for surgical applications.

Indeed, the use of compliant joints is a very promising avenue for the delevopment of compact underactuated grippers, even if their theoretical analysis is still in its infancy (Birglen, 2006). Important challenges can be mentioned like stability of grasping (Birglen et al., 2008; Kragten et al., 2008) or force-isotropic properties (Krut, 2005), and the use of compliant joints greatly increases the complexity of the corresponding analyses.

Yet, the present paper does not address these issues. Its objective is merely to illustrate the use of the KSM and the QSM in the modelling of UMs - and in particular underactuated fingers, in order to show the possibilities offered by these models. It is argued that, with further investigations, these models - especially the generalized stiffness matrix (generalized SM) (Cho et al., 1989; Griffis and Duffy, 1991; Quennouelle and Gosselin, 2008) and the transmission matrix can become valuable tools for the analysis and control of underactuated robotic hands. 


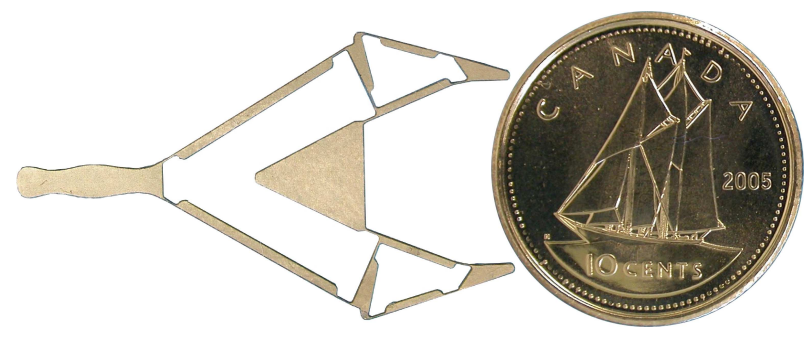

Figure 1. Sub-centimetre underactuated compliant gripper (Boudreault and Gosselin, 2006).

The first section of this paper deals with the theoretical formulation of the KSM and the QSM. The equivalence between the general formulation of the static equilibrium applied to UMs and the kinetostatic relation between the actuation torques and the contact forces used in the underactuation theory is demonstrated. For that, the models have been extended to the case of dependent actuated joints. Then, when all external forces are function of the configuration of the mechanism, the system $\{\mathrm{UM}+$ grasped object $\}$ becomes equivalent to a fully actuated mechanism. In the second section, the KSM and the QSM are applied to a compliant 2DOF underactuated finger. The implementation of the simulation is presented and some results are discussed. The contributions of the KSM and the QSM to this kind of application are underlined for analysis, design and control, respectively.

\section{Kinematostatic modelling of underactuated mechanisms}

\subsection{Concept of kinematostatic modelling}

\subsubsection{Kinematic model}

The kinematic constraints between the dependent coordinates $\lambda$, the pose of the end-effector platform $\boldsymbol{x}$ and the independent generalized coordinates $\psi$ are written as $\mathcal{K}(\boldsymbol{\lambda}, \boldsymbol{x}, \boldsymbol{\psi})=$ 0. By solving the latter, the kinematic model can be written as a function of the independent generalized coordinates: $\boldsymbol{x}=\boldsymbol{P}(\psi)$ for the Cartesian pose and $\boldsymbol{\lambda}=\boldsymbol{G}(\boldsymbol{\psi})$ for the dependent coordinates. The degree of mobility of the mechanism is noted $\mathfrak{M}$ and the number of actuators is noted $\mathfrak{A}$.

\subsubsection{Static model}

This model is less commonly used, (it is sometimes called kinetostatic). The static constraints between the external loads $\boldsymbol{f}$, the commanded position of the actuators $\phi_{0}$ and the configuration of the mechanism, represented by the generalized coordinates are written as $\mathcal{S}\left(\psi, f, \phi_{0}\right)=\mathbf{0}$. By solving these static constraints, the configuration of the mechanism can be written as a function of the external loads and the position of the actuators: $\boldsymbol{\psi}=\mathcal{F}\left(\boldsymbol{f}, \boldsymbol{\phi}_{0}\right)$.

\subsubsection{Kinematostatic model}

By solving simultaneously the kinematic and the static constraints, the pose of the end-effector of a mechanism can be written as a function of the external parameters only. This is written as

$\boldsymbol{x}=\mathcal{M}\left(f, \phi_{0}\right)=\mathcal{P}\left(\mathcal{F}\left(f, \phi_{0}\right)\right)$.

\subsection{General formulation of the static equilibrium}

When the gravitational loads are not taken into account, the equation describing the static equilibrium in a parallel mechanism is written as

$\mathcal{S}\left(\psi, \boldsymbol{f}, \phi_{0}\right)=\tau_{\psi}+\mathbf{G}^{T} \tau_{\lambda}-\mathbf{J}^{T} \boldsymbol{f}=\mathbf{0}$,

where $\tau_{\psi}$ and $\tau_{\lambda}$ are the forces applied on the corresponding joints and $\mathbf{G}=d \boldsymbol{\lambda} / d \boldsymbol{\psi}$ and $\mathbf{J}=d \boldsymbol{\lambda} / d \boldsymbol{\psi}$ are the Jacobian matrices of the vectors of the dependent coordinates and of the pose of the end-effector with respect to the generalized coordinates.

\subsection{Theory of underactuation}

In Birglen et al. (2008), the relation between the contact force $\boldsymbol{f}_{c}$ of a grasping UM and the torque $\boldsymbol{\tau}_{\phi}$ provided by its actuators is expressed as

$\mathbf{J}_{c}^{\star} \boldsymbol{f}_{c}=\mathbf{T}_{u}^{\star} \boldsymbol{t}$,

with $\mathbf{J}_{c}^{\star}=\mathbf{J}_{c}^{T}$, such that $\mathbf{J}_{c}^{\star} \boldsymbol{f}_{c}$ corresponds to the projection of the effect of the contact forces, into the generalized coordinates domain; and $\mathbf{T}_{u}^{\star}=\mathbf{T}_{u}^{-T}$, such that $\mathbf{T}_{u}^{\star} \boldsymbol{t}$ corresponds to the projection of the torques provided by the actuator and the springs in the independent joints, into the generalized coordinates domain.

With the following assumptions, the general formulation of the static equilibrium (Eq. 2) can be brought to the particular case given in Eq. (3):

- The vector containing the position of all contact points is noted $\boldsymbol{x}_{c}^{T}=\left[\boldsymbol{x}_{p}^{T} ; \boldsymbol{x}_{q}^{T} ; \ldots\right]$, and the corresponding Jacobian matrix is : $\mathbf{J}_{c}^{T}=\left[\mathbf{J}_{p}^{T} ; \mathbf{J}_{q}^{T} ; \ldots\right]$.

- The vector containing the components of all contact forces is $\boldsymbol{f}_{c}^{T}=\left[\boldsymbol{f}_{p}^{T} ; \boldsymbol{f}_{q}^{T} ; \ldots\right]$. Therefore, the generalized forces due to the contact forces are written as $-\mathbf{J}_{c}^{T} \boldsymbol{f}_{c}=$ $-\mathbf{J}_{p}^{T} \boldsymbol{f}_{p}-\mathbf{J}_{q}^{T} \boldsymbol{f}_{q}-\ldots$

- The actuated joints are not considered as independent coordinates, i.e., the corresponding coordinates $\phi$ are components of vector $\lambda$. The unactuated dependent coordinates are noted $\lambda_{N}$. They have zero stiffness and therefore cannot resist or transmit any force in a static configuration. One has $\boldsymbol{\tau}_{\lambda}^{T}=\left[\boldsymbol{\tau}_{\phi}^{T} ; \mathbf{0}^{T}\right]$. 
- Matrix $\mathbf{H}$ is composed of the rows of $\mathbf{G}$ corresponding to the actuated dependent joints and $\mathbf{N}$ is composed of the rows of $\mathbf{G}$ corresponding to the unactuated dependent joints, such that $\mathbf{G}^{T} \boldsymbol{\tau}_{\lambda}=\mathbf{H}^{T} \boldsymbol{\tau}_{\phi}+\mathbf{N}^{T} \mathbf{0}=\mathbf{H}^{T} \boldsymbol{\tau}_{\phi}$.

- Among the $\mathfrak{M}$ generalized coordinates, $\mathfrak{A}$ have a zero stiffness and the $\mathfrak{L}$ others, noted $\psi_{K}$, have a finite stiffness $(\mathfrak{L}=\mathfrak{M}-\mathfrak{A})$. By defining $\mathbf{L}=\left[\mathbf{0}_{\mathfrak{L} \times \mathfrak{A}} ; \mathbf{1}_{\mathfrak{L} \times \mathfrak{L}}\right]$, the torques applied to the independent joints are written as $\boldsymbol{\tau}_{\psi}=\left[\mathbf{0}_{\mathfrak{A}}^{T} ; \boldsymbol{\tau}_{\psi_{K}}^{T}\right]^{T}=\mathbf{L}^{T} \boldsymbol{\tau}_{\psi_{K}}$.

- The vector of joint torques, used in Birglen et al. (2008), is $\boldsymbol{t}^{T}=\left[\boldsymbol{\tau}_{\phi}^{T} ; \boldsymbol{\tau}_{\psi_{K}}^{T}\right]$.

- In Birglen et al. (2008), matrix $\mathbf{T}_{u}$ is the Jacobian of vector $\boldsymbol{\psi}$ with respect to vector $\left[\boldsymbol{\phi}^{T} ; \boldsymbol{\psi}_{K}^{T}\right]^{T}$. It inverse is given by

$$
\mathbf{T}_{u}^{-T}=\left[\left(\frac{d \boldsymbol{\phi}}{d \boldsymbol{\psi}}\right)^{T} ;\left(\frac{d \boldsymbol{\psi}_{K}}{d \boldsymbol{\psi}}\right)^{T}\right]=\left[\mathbf{H}^{T} ; \mathbf{L}^{T}\right] .
$$

Matrices $\mathbf{H}$ and $\mathbf{l}$ being respectively of dimension $\mathfrak{M} \times \mathfrak{A}$ and $\mathfrak{M} \times \mathfrak{L}$, matrix $\mathbf{T}_{u}^{-1}$ is a square and invertible $\mathfrak{M} \times \mathfrak{M}$ matrix.

Thus, the static equilibrium (Eq. 2) is equivalent to

$\mathcal{S}=\mathbf{L}^{T} \boldsymbol{\tau}_{\psi_{K}}+\mathbf{H}^{T} \boldsymbol{\tau}_{\phi}-\mathbf{J}_{c}^{T} \boldsymbol{f}_{c}=\mathbf{T}_{u}^{-T} \boldsymbol{t}-\mathbf{J}_{c}^{T} \boldsymbol{f}_{c}=\mathbf{0}$.

This confirms that Eq. (3) is a particular case of the general formulation of the static equilibrium of a parallel mechanism.

\subsection{Concept of underactuation}

For the reasons mentioned above, the KSM of an UM can be easily established. In the case of a grasping mechanism, there is no pose of end-effector platform to be calculated, but a configuration $\psi$ to be determined as a function of the positions of the actuators and of the contact forces, written as $\boldsymbol{\psi}=\mathcal{F}\left(\phi_{0}, \boldsymbol{f}_{c}\right)$.

In practice, such a mechanism is called underactuated because there are $\mathfrak{M}$ degrees of mobility for only $\mathfrak{A}$ actuators. But in this theoretical KSM, it can be seen that, not only the actuators have an impact on the configuration of the mechanism, but also the $3 \times n$ components of the contact forces.

If these external forces are considered as additional actuators acting on the mechanism, and if the $3 \times n$ components of the external forces are reduced to only $\mathfrak{L}$ independent components chosen among them and noted $f$, the KSM becomes the one of a classical fully actuated mechanism: $\psi=\mathcal{F}\left(\phi_{0}, f\right)$.

\subsection{Quasi-static modelling}

\subsubsection{Linear relationship}

The QSM of a parallel mechanism provides linear relationships between the infinitesimal variation of the configuration $\psi$ and that of the external parameters ( $\phi_{0}$ and $f$ ) (Quennouelle and Gosselin, 2009). This is written as

$d \boldsymbol{\psi}=\mathbf{T}_{0} d \boldsymbol{\phi}_{0}+\mathbf{K}_{M_{0}}^{-1} \mathbf{J}^{T} d \boldsymbol{f}$, where $\mathbf{T}_{0}$ is the transmission matrix and $\mathbf{K}_{M_{0}}$ is the generalized SM where the external loads are independent from the configuration of the mechanism (Quennouelle and Gosselin, 2008). One has

$\mathbf{K}_{M_{0}}=\mathbf{K}_{\psi}+\mathbf{K}_{I}+\mathbf{K}_{E_{0}}$,

with $\mathbf{K}_{\psi}$ the SM due to the independent joints, $\mathbf{K}_{I}$ the internal SM (due to the stiffness of the dependent joints and the internal forces) and $\mathbf{K}_{E_{0}}$ is the SM due to the external loads (independent from $\psi$ ).

\subsubsection{Transmission matrix}

The transmission matrix is a $\mathfrak{M} \times \mathfrak{A}$ matrix that maps the effects of a variation in the commanded value of the actuators onto the generalized coordinates, taking into account the effects of the compliance due to the contact forces. It is different from the kinematic "transmission matrix" used in the theory of underactuation (Birglen et al., 2008), because only the former considers the compliance of the joints.

In Quennouelle and Gosselin (2009), only the case for which the actuated coordinates are independent is addressed. In the present case, the actuated joints are considered as dependent coordinates and the transmission matrix $\mathbf{T}_{0}$ is then defined as

$\mathbf{T}_{0}=\frac{\partial \boldsymbol{\psi}}{\partial \boldsymbol{\phi}_{0}}=-\left(\frac{d \mathcal{S}}{d \boldsymbol{\psi}}\right)^{-1} \frac{d \mathcal{S}}{d \boldsymbol{\phi}_{0}}=\mathbf{K}_{M_{0}}^{-1} \mathbf{H}^{T} \mathbf{K}_{\phi}$.

When the actuated joints are dependent in Eq. 2), only the term $\mathbf{G}^{T} \boldsymbol{\tau}_{\lambda}$, and in particular $\mathbf{H}^{T} \boldsymbol{\tau}_{\phi}$, are function of $\boldsymbol{\phi}_{0}$. One has

$\frac{d \mathcal{S}}{d \boldsymbol{\phi}_{0}}=\frac{d\left(\mathbf{H}^{T} \boldsymbol{\tau}_{\phi}\right)}{d \boldsymbol{\phi}_{0}}=\left(\frac{d \mathbf{H}^{T}}{d \boldsymbol{\phi}_{0}} \tau_{\phi}\right)-\mathbf{H}^{T} \mathbf{K}_{\phi}=-\mathbf{H}^{T} \mathbf{K}_{\phi}$,

with $\left(d \mathbf{H}^{T} / d \boldsymbol{\phi}_{0}\right) \tau_{\phi}=\mathbf{0}$ because $\boldsymbol{\psi}$, the configuration of the mechanism, is not directly (kinematically) function of $\boldsymbol{\phi}_{0}$.

\subsubsection{Effect of the external loads}

Independent: In an UM used as a gripper, the external loads are the contact forces. When these forces are considered as independent from the configuration of the mechanism, they are taken into account through the $\mathbf{K}_{E_{0}}$ matrix as follows:

$\mathbf{K}_{E_{0}}=-\left(\frac{d \mathbf{J}^{T}}{d \boldsymbol{\psi}}\right) \boldsymbol{f}$.

Therefore a contact force on one phalanx can modify the stiffness of the generalized coordinates and, in turn, the variation of the configuration of the mechanism due to a variation of the external parameters $\left(\phi_{0}\right.$ and $\left.\boldsymbol{f}\right)$.

Dependent: However, in a context of grasping, the contact forces are generally not independent from the configuration of the mechanism, since they are mainly generated by the 
pressure of the phalanges on the grasped object. Therefore, these contact forces are in turn, function of the only truly independent external parameters, namely, the commanded position of the actuators. The contact force applied on an object is related to the position of the phalanx through a SM, $\mathbf{K}_{c}=\operatorname{diag}\left(\mathbf{K}_{p} ; \mathbf{K}_{q} ; \ldots\right)$, each $\mathbf{K}_{i}$ being the SM of the object at the $i^{\text {th }}$ contact point. The variation of the force is given by $d \boldsymbol{f}=\mathbf{K}_{c} d \boldsymbol{x}=\mathbf{K}_{c} \mathbf{J} d \boldsymbol{\psi}$.

Then, the external SM $\mathbf{K}_{E}$ is expressed as

$\mathbf{K}_{E}=-\left(\frac{d \mathbf{J}^{T}}{d \boldsymbol{\psi}}\right) \boldsymbol{f}-\mathbf{J}^{T} \mathbf{K}_{c} \mathbf{J}=\mathbf{K}_{E_{0}}-\mathbf{J}^{T} \mathbf{K}_{c} \mathbf{J}$.

A generalized SM $\mathbf{K}_{M}$ can be defined that includes this stiffness due to the variation of the external loads. One has

$\mathbf{K}_{M}=\mathbf{K}_{M 0}-\mathbf{J}^{T} \mathbf{K}_{c} \mathbf{J}$.

Thus, the QSM becomes simply function of the $\mathfrak{A}$ commanded positions of the actuators. One has

$d \boldsymbol{\psi}=\mathbf{K}_{M}^{-1} \mathbf{K}_{\phi}^{\star} d \boldsymbol{\phi}_{0}=\mathbf{T} d \boldsymbol{\phi}_{0}$.

In the latter equation, $\mathbf{T}\left(=d \boldsymbol{\psi} / d \boldsymbol{\phi}_{0}\right)$ is no longer a partial derivative. This is equivalent to saying that when the mechanism is grasping a compliant object, the degree of mobility is reduced from $\mathfrak{M}$ to $\mathfrak{A}$.

\section{Application to a 2-DOF compliant finger}

\subsection{2-DOF compliant underactuated finger}

The KSM and the QSM of compliant mechanisms are applied to a compliant finger shown in Fig. 2a (more details about its design are presented in Boudreault and Gosselin (2006)).

A schematic representation of the mechanism is given in Fig. 2b. The lengths (in mm) of the links are $e=6, h=54$, $g=10, b=8, c=54, n=30, l_{x}=25, l_{y}=20, d_{1}=10$ and $d_{2}=9$. The angle $\phi=134.5 \mathrm{deg}$. The actuator is not modelled as a simple torque $T_{\rho}$ as it is often the case in the literature, but - according to the reality - as a prismatic compliant actuator of variable commanded length $\rho_{0}$ and of actual length $\rho$. The contact forces $\boldsymbol{f}_{p}$ and $\boldsymbol{f}_{q}$ on the two phalanges are respectively applied at a distance $l_{p}$ and $l_{q}$ of the revolute joints $\theta_{p}$ and $\theta_{q}$.

\subsection{Symbolic modelling}

The mechanism has eight joints with coordinates: $\theta_{p}, \theta_{1}, \theta_{2}$, $\theta_{3}, \theta_{4}, \theta_{5}, \theta_{6}$ and $\rho\left(\theta_{q}\right.$ being rigidly linked to $\left.\theta_{2}\right)$ and two planar kinematic loops, thus it has two degrees of mobility. The chosen independent generalized coordinates are $\psi^{T}=\left[\theta_{p} ; \theta_{1}\right]$ and the dependent ones are assembled in $\lambda^{T}=\left[\theta_{2} ; \theta_{3} ; \theta_{4}\right]$ for the four-bar linkage and $\rho$ for the actuated loop (actually joints $\theta_{5}$ and $\theta_{6}$ are assumed to be conventional, i.e., with a zero stiffness, and therefore their determination is not useful in the KSM). The position of the final phalanx is given by $\theta_{q}=\theta_{2}+\phi-\pi$.

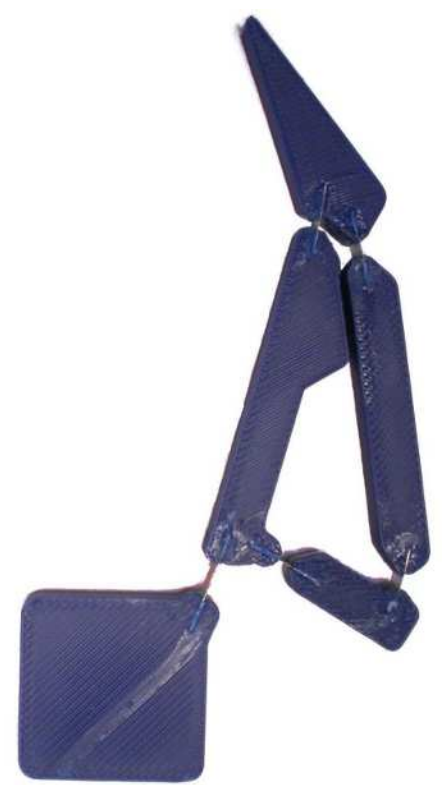

(a) Prototype with joints made of nitinol (Boudreault and Gosselin, 2006).

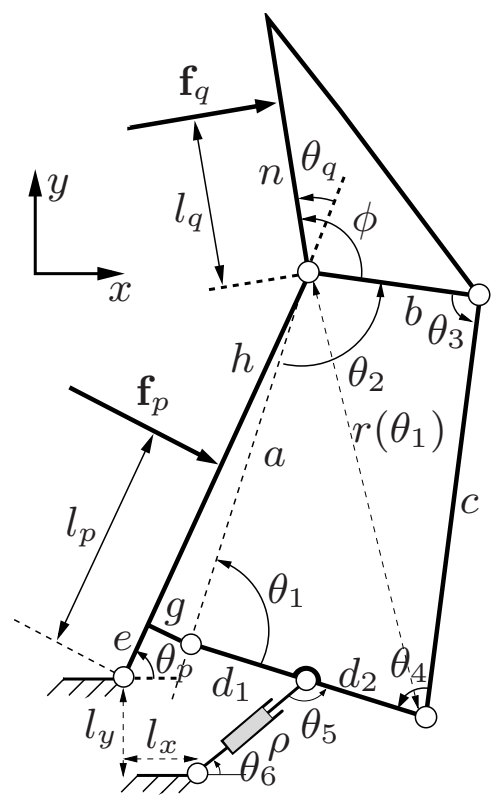

(b) Geometry of the 2-DOF underactuated finger.

Figure 2. 2-DOF compliant underactuated finger.

\subsubsection{Kinematic model of the four-bar linkage}

This passive loop defines three kinematic constraints that have to be satisfied by the dependent coordinates. Using two constant lengths $a=\sqrt{h^{2}+g^{2}}$ and $d=d_{1}+d_{2}$ and a variable length $r=\sqrt{a^{2}+d^{2}-2 a d \cos \theta_{1}}$, the solution to these constraints, corresponding to the configuration shown in Fig. 2b, 
is

$$
\left\{\begin{array}{l}
\theta_{2}=\arccos \left(\frac{r^{2}+a^{2}-d^{2}}{2 a r}\right)+\arccos \left(\frac{b^{2}+r^{2}-c^{2}}{2 b r}\right), \\
\theta_{3}=\arccos \left(\frac{b^{2}+c^{2}-r^{2}}{2 b c}\right), \\
\theta_{4}=\arccos \left(\frac{r^{2}+c^{2}-b^{2}}{2 c r}\right)+\arccos \left(\frac{d^{2}+r^{2}-a^{2}}{2 d r}\right) .
\end{array}\right.
$$

A simple analysis of the workspace of the four-bar mechanism, in the configuration shown in Fig. 2b, reveals that these relations are only valid for $0.9818 \leq \theta_{1} \leq 1.8177$.

Instantaneous kinematic constraints: The differentiation of the kinematic constraints with respect to the joint coordinates can be written as $\mathbf{S}_{\theta 1} d \theta_{1}=-\mathbf{S}_{\lambda} d \lambda$, with

$\mathbf{S}_{\psi}=\left[\begin{array}{cc}0 & -a s_{1}+b s_{12}-c s_{123} \\ 0 & a c_{1}-b c_{12}+c c_{123} \\ 0 & 1\end{array}\right] ; \mathbf{S}_{\lambda}=\left[\begin{array}{ccc}b s_{12}-c s_{123} & -c s_{123} & 0 \\ -b c_{12}+c c_{123} & c c_{123} & 0 \\ 1 & 1 & 1\end{array}\right]$

where $s_{i \ldots k}$ stands for $\sin \left(\theta_{i}+\cdots+\theta_{k}\right.$ ) ( $c$ for $\cos$ respectively). From the latter equation, matrix $\mathbf{G}$ is obtained as $\mathbf{G}=-\mathbf{S}_{\lambda}^{-1} \mathbf{S}_{\psi}$.

\subsubsection{Kinematic model of the actuation loop}

The actuated loop is shown in details in Fig. 3a and the following constant geometric parameters are introduced: $l=\sqrt{l_{x}^{2}+l_{y}^{2}}, \quad r_{1}=\sqrt{e^{2}+g^{2}}, \quad \alpha=\arctan (g / e), \quad \beta=\pi-\alpha-$ $\arctan (g / h), \gamma=(\pi / 2)-\arctan \left(l_{x} / l_{y}\right)$. The finger is actuated by a prismatic actuator that moves the link $d$. This actuator has a finite stiffness, therefore it is modelled as an ideal prismatic actuator $\rho_{0}$ connected in series with a linear spring of stiffness $k_{\rho}$. The commanded position is $\rho_{0}$ and the actual position of this compliant actuator is $\rho$.

By satisfying the loop-closure constraints and introducing $\theta_{p}^{\prime}=\theta_{p}-\alpha+\gamma$ and $\theta_{1}^{\prime}=\theta_{1}-\beta$, the actuated coordinate $\rho$ can be written as a function of the generalized coordinates:

$\rho=\sqrt{r_{1}^{2}+d_{1}^{2}+l^{2}+2 d_{1} r_{1} c_{1}^{\prime}-2 r_{1} l c_{p}^{\prime}-2 d_{1} l \cos \left(\theta_{p}^{\prime}-\theta_{1}^{\prime}\right)}$.

And the Jacobian matrix $\mathbf{H}$ can be calculated as

$\mathbf{H}^{T}=\left[\begin{array}{c}\frac{\partial \rho}{\partial \theta_{p}} \\ \frac{\partial \rho}{\partial \theta_{1}}\end{array}\right]=\left[\begin{array}{r}\frac{l}{\rho}\left(r_{1} \sin \theta_{p}^{\prime}+d_{1} \sin \left(\theta_{p}^{\prime}-\theta_{1}^{\prime}\right)\right) \\ -\frac{d_{1}}{\rho}\left(r_{1} \sin \theta_{1}^{\prime}+l \sin \left(\theta_{p}^{\prime}-\theta_{1}^{\prime}\right)\right)\end{array}\right]$.

\subsubsection{Contact points}

In this paper, it is assumed that the grasped object is fixed, such that the effect of the force applied by the finger is to deform the object and not to displace it. It is also assumed that the first contact between the object and the finger is on the first phalanx, and then the second contact point is on the second phalanx (see Fig. 3b). The design of the finger and

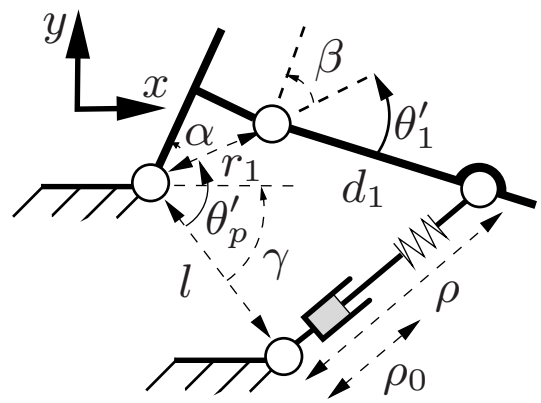

(a) Actuation loop.

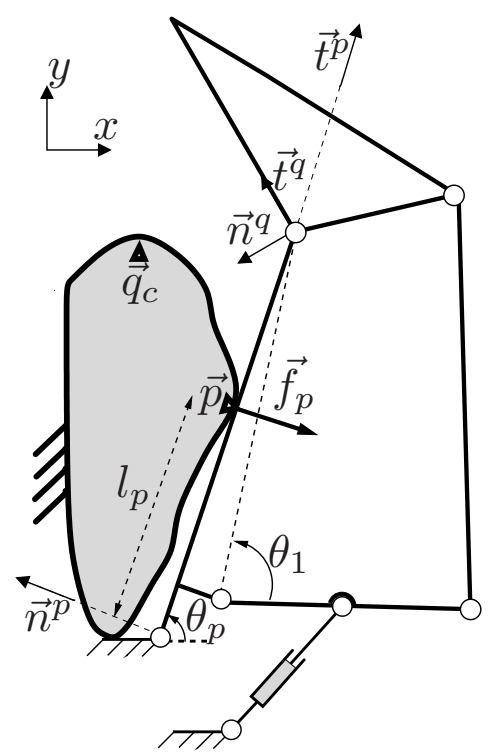

(b) Finger grasping a compliant object.

Figure 3. 2-DOF compliant underactuated finger.

the position of the second contact point are chosen to prevent any ejection, i.e., the first contact cannot be lost when the second contact is established.

The first contact is established in $\boldsymbol{p}_{c}=\left[x_{p c} ; y_{p c}\right]^{T}$ and the second one in $\boldsymbol{q}_{c}=\left[x_{q c} ; y_{q c}\right]^{T}$. After the deformation of the object, the actual positions of the contact points become $\boldsymbol{x}_{p}$ and $\boldsymbol{x}_{q}$ and the corresponding deformations are noted $\delta \boldsymbol{x}_{p}=$ $\boldsymbol{x}_{p}-\boldsymbol{p}_{c}$ and $\delta \boldsymbol{x}_{q}=\boldsymbol{x}_{q}-\boldsymbol{q}_{c}$. The contact points correspond to the following particular configurations of the mechanism:

- the first contact is established when $\theta_{p}$ is greater than $\theta_{p c}=\arctan \left(y_{p c}, x_{p c}\right)$,

- then, the second contact is established when $\theta_{q}$ is greater than $\theta_{q c}=\arctan \left(y_{q c}-m s_{p}, x_{q c}-m c_{p}\right)$.

Finally, $\delta \boldsymbol{x}_{p}$ and $\delta \boldsymbol{x}_{q}$ are equal to $\mathbf{0}$ when the corresponding contact is not established. 
Assuming that the position of a contact point relatively to the corresponding phalanx is constant, i.e., $l_{p}$ and $l_{q}$ are constant, the position of the contact points are given by

$\boldsymbol{x}_{p}=\left[\begin{array}{l}l_{p} c_{p} \\ l_{p} s_{p}\end{array}\right]$ and $\boldsymbol{x}_{q}=\left[\begin{array}{c}m c_{p}+l_{q} c_{p q} \\ m s_{p}+l_{q} s_{p q}\end{array}\right]$,

with $m=h+e, l_{q}=\sqrt{\left(x_{q c}-m c_{p}^{q}\right)^{2}+\left(y_{q c}-m s_{p}^{q}\right)^{2}}$ and $l_{p}=$ $\sqrt{x_{p c}^{2}+y_{p c}^{2}}, \theta_{p}^{q}$ is the value of $\theta_{p}$ when the second contact is established. The mapping between the global frame and the local frames $\left(\boldsymbol{t}^{i}, \boldsymbol{n}^{i}\right)$ attached to each phalanx is performed through rotation matrices $\mathbf{Q}_{i}$, written as

$\mathbf{Q}_{p}=\left[\begin{array}{rr}c_{p} & s_{p} \\ -s_{p} & c_{p}\end{array}\right]$ and $\mathbf{Q}_{q}=\left[\begin{array}{cc}c_{p q} & s_{p q} \\ -s_{p q} & c_{p q}\end{array}\right]$

In these frames, the contact points are given by $\boldsymbol{x}_{p}^{p}=\mathbf{Q}_{p} \boldsymbol{x}_{p}=$ $\left[l_{p} ; 0\right]^{T}$ and $\boldsymbol{x}_{q}^{q}=\left[m c_{p}+l_{q} ;-m s_{p}\right]^{T}$, and their corresponding Jacobian matrices are given by

$\mathbf{J}_{p}^{p}=\mathbf{Q}_{p} \frac{d \boldsymbol{x}_{p}}{d \boldsymbol{\psi}}=\left[\begin{array}{ll}0 & 0 \\ l_{p} & 0\end{array}\right]$ and $\mathbf{J}_{q}^{q}=\left[\begin{array}{cc}m s_{q} & 0 \\ m c_{q}+l_{q} & l_{q} \eta\end{array}\right]$

where $\eta$ relates the variation of angle $\theta_{q}$ to the variation of $\theta_{1}$. It is written as $\eta=d \theta_{q} / d \theta_{1}=\left(a s_{23}-b s_{3}\right) / b s_{3}$.

\subsubsection{Static model}

Torques/forces in the joints: In this compliant mechanism actuated by a compliant actuator, all the joints can be subjected to a torque/force, even in a static configuration. Since, the stiffness of the joints is supposed to be constant, these torques/forces are written $\boldsymbol{\tau}_{v}=\mathbf{K}_{v}\left(\boldsymbol{v}-\boldsymbol{v}_{0}\right.$ ), (for $v=\psi, \lambda, \rho$ ). Matrices $\mathbf{K}_{v}$ is diagonal and contains the stiffnesses of the corresponding joints, $v_{0}$ is the vector composed by the undeformed value of the joints.

Contact forces: Since the ceased object is elastic, the contact forces can be written as a function of the contact points and in turn, of the mechanism configuration. Both contact forces are assumed to be proportional to the deformation of the object, i.e., the latter is supposed to have a constant and isotropic SM (this assumption being reasonable for relatively small deformations). Obviously the contact force is zero when there is no contact (so is $\delta \boldsymbol{x}_{i}$ ). Each force is expressed in the local frame as

$\boldsymbol{f}_{i}^{i}=-\mathbf{K}_{i} \delta \boldsymbol{x}_{i}^{i}=-\mathbf{K}_{i} \mathbf{Q}_{i} \delta \boldsymbol{x}_{i}=\left[\begin{array}{cc}-k_{i}( & \left.c_{i} \delta x_{i}+s_{i} \delta y_{i}\right) \\ -k_{i}\left(-s_{i} \delta x_{i}+c_{i} \delta y_{i}\right)\end{array}\right]=\left[\begin{array}{c}f_{t i} \\ f_{n i}\end{array}\right]$.

In order to ease a future study of the stability of the grasp, the tangential components of all forces can be assembled in a vector $f_{t}$ and the normal components in a vector $f_{n}$ (see Birglen et al., 2008). One has $\boldsymbol{f}_{t}=\left[f_{t p} ; f_{t q}\right]^{T}$ and $\boldsymbol{f}_{n}=\left[f_{n p} ; f_{n q}\right]^{T}$.
The rows of the Jacobian matrices $\mathbf{J}_{p}^{p}$ and $\mathbf{J}_{q}^{q}$ can be reassembled into two matrices $\mathbf{J}_{t}$ and $\mathbf{J}_{n}$ (respectively named $\mathbf{J}_{2}$ and $\mathbf{J}_{1}$ in Birglen et al. (2008)). These matrices are

$\mathbf{J}_{t}=\left[\begin{array}{cc}0 & 0 \\ m s_{q} & 0\end{array}\right]$ and $\mathbf{J}_{n}=\left[\begin{array}{cc}l_{p} & 0 \\ m c_{q}+l_{q} & l_{q} \eta\end{array}\right]$.

Static equilibrium: In this finger, the static equilibrium of the mechanism is written as

$\mathcal{S}\left(\psi, \rho_{0}\right)=\boldsymbol{\tau}_{\psi}+\mathbf{H}^{T} \tau_{\rho}+\mathbf{G}^{T} \boldsymbol{\tau}_{\lambda}-\mathbf{J}_{n}^{T} \boldsymbol{f}_{n}-\mathbf{J}_{t}^{T} \boldsymbol{f}_{t}=\mathbf{0}$.

Ejection of the finger: The static equilibrium given in Eq. (21) can only be reached for positive values of $\delta n_{i}$, i.e., negatives values of the normal contact forces $\left(f_{n i}=-k_{i} \delta n_{i}\right)$. Therefore, the following conditions have to be satisfied:

$\left\{\begin{array}{l}f_{n p} \geq 0 \\ f_{n q} \geq 0\end{array}\right.$ with $\left[\begin{array}{l}f_{n p} \\ f_{n q}\end{array}\right]=\mathbf{J}_{n}^{-T}\left(\boldsymbol{\tau}_{\psi}+\mathbf{H}^{T} \tau_{\rho}+\mathbf{G}^{T} \boldsymbol{\tau}_{\lambda}-\mathbf{J}_{t}^{T} \boldsymbol{f}_{t}\right)$.

\subsubsection{Stiffness matrices}

Joint stiffness: The SM due to the dependent coordinates in the generalized space is given by

$\mathbf{K}_{I}=\frac{d\left(\mathbf{G}^{T} \boldsymbol{\tau}_{\lambda}\right)}{d \boldsymbol{\psi}}=\frac{d\left(\mathbf{G}^{T} \boldsymbol{\tau}_{\lambda}\right)}{d \boldsymbol{G}} \mathbf{G}$, with $\boldsymbol{\tau}_{\lambda}=\mathbf{K}_{\lambda}\left(\lambda-\lambda_{0}\right)$.

In this matrix, only the bottom-right coefficient is not zero, i.e., the stiffness of the dependent joints affects only the position of the second phalanx $\theta_{q}$.

Active stiffness due to contact forces: When the corresponding contact is established, its effects on the stiffness of the mechanism are given by

$\mathbf{K}_{E t}^{T}=\left[\begin{array}{cc}k_{q} m s_{q}\left(s_{p q} x_{p c}-c_{p q} y_{p c}\right) & 0 \\ \left(-m c_{q} f_{t q}+k_{q} m s_{q}\left(-m s_{q}+s_{p q} x_{p c}-c_{p q} y_{p c}\right)\right) \eta & 0\end{array}\right]$

$\mathbf{K}_{E n}=\left[\begin{array}{ll}D & E \\ F & G\end{array}\right]$, where the components are

$$
\begin{aligned}
& D=l_{p} k_{p}\left(c_{p} x_{p c}+s_{p} y_{p c}\right)+k_{q}\left(m c_{q}+l_{q}\right)\left(c_{p q} x_{q c}+s_{p q} y_{q c}\right), \\
& E=-\left(-m s_{q} f_{n q}+k_{q}\left(m c_{q}+l_{q}\right)\left(m c_{q}-c_{p q} x_{q c}-s_{p q} y_{q c}\right)\right) \eta, \\
& F=l_{q} k_{q}\left(c_{p q} x_{q c}+s_{p q} y_{q c}\right) \eta,
\end{aligned}
$$

$G=-l_{q} k_{q}\left(m c_{q}-c_{p q} x_{q c}-s_{p q} y_{q c}\right) \eta^{2}-l_{q} f_{n q} \frac{a s_{2}\left(c+a c_{23}-b c_{3}\right)}{b c s_{3}^{2}}$

Active stiffness due to the compliant actuator:

$\mathbf{K}_{\rho}=\left(\frac{d\left(\mathbf{H}^{T} \tau_{\rho}\right)}{d \boldsymbol{\psi}}\right)=\left[\frac{\partial^{2} \rho}{\partial \boldsymbol{\psi}^{2}}\right] \tau_{\rho}+\mathbf{H}^{T} k_{\rho} \mathbf{H}$.

\subsubsection{Quasi-static model}

The generalized SM is given by $\mathbf{K}_{M}=\mathbf{K}_{\psi}+\mathbf{K}_{I}+\mathbf{K}_{\rho}+\mathbf{K}_{E t}+$ $\mathbf{K}_{E n}$ and the matrix of transmission ratio is calculated as $\mathbf{T}=$ $\mathbf{K}_{M}^{-1} \mathbf{K}_{\rho 0}^{\star}$. 


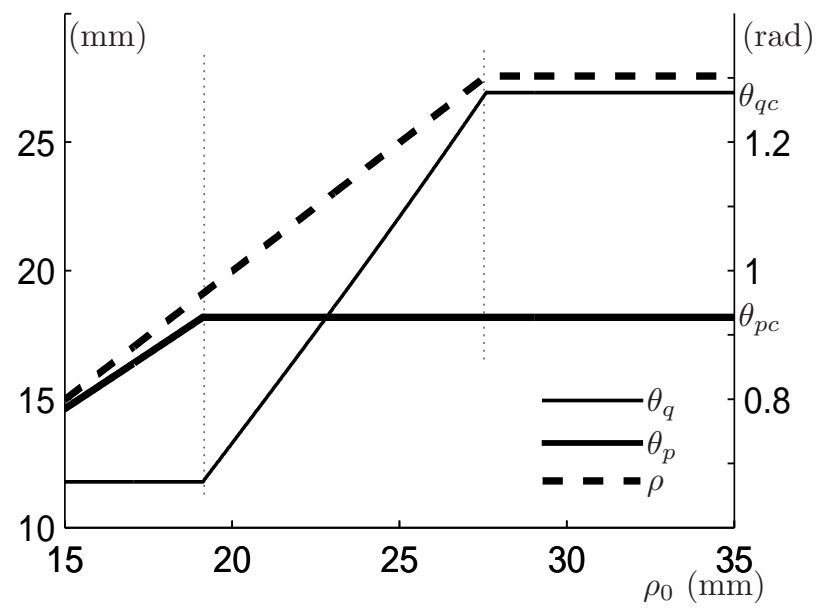

Figure 4. Configuration of the mechanism as a function of $\rho_{0}$.

\subsection{Numerical simulations}

\subsubsection{Management of the contact}

The simulation (using the KSM or the QSM) starts with the finger open and the contact with the object not established, then the value of the only commandable parameter, i.e., $\rho_{0}$, is modified. At each step of the simulation, the corresponding values of the stiffness used for the computation are determined as follows: if $\theta_{p} \geq \theta_{p c}$ (first contact established), $k_{p} \leftarrow k_{c}$ else $k_{p} \leftarrow 0$. And if $\theta_{p} \geq \theta_{p c}$ and $\theta_{q} \geq \theta_{q c}$ (both contacts established), then $k_{q} \leftarrow k_{c}$ otherwise $k_{q} \leftarrow 0$. It is also assumed that the increments are small enough to neglect the effect of a contact established between two steps of computation.

Since when $k_{i}=0$, the corresponding contact force $\boldsymbol{f}_{i}$ and SM $\mathbf{K}_{E i}$ are zero for any value of $\delta \boldsymbol{x}_{i}$, the calculation can be directly performed with the relations given in the previous section.

\subsubsection{Kinematostatic simulation}

Simulation: By solving numerically the static constraints, the configuration of the mechanism can be determined as a function of the commanded value of the actuator $\rho_{0}$ only. Even if the computational time of the KSM depends on the gap between the initial configuration (used to start the numerical method) and the found configuration, it is relatively fast, about $10 \mathrm{~ms}$ in our Matlab program. This model provides exact results and is suitable for the analysis and design of a UM.

The undeformed angles of the compliant joints are (in $\mathrm{rad}$ ): $\theta_{p 0}=\pi / 4, \theta_{10}=\pi / 2, \theta_{20}=1.437, \theta_{30}=1.819$, $\theta_{40}=1.457$. The positions of the objects are (in $\mathrm{mm}$ ) $p_{x}=15, p_{y}=20, q_{x}=15, q_{y}=65$.

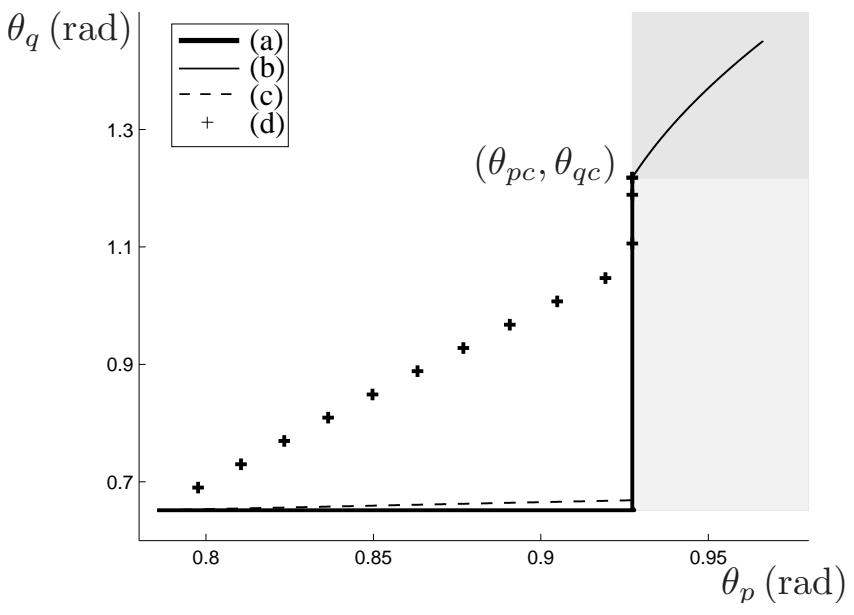

Figure 5. Configurations of several mechanisms in the $\left(\theta_{p}, \theta_{q}\right)$ plane.

Results: Figure 4 shows the configuration $\left(\theta_{p}, \theta_{q}\right)$ and the actual length of the actuator for $\rho_{0}$ between $15 \mathrm{~mm}$ and $35 \mathrm{~mm}$. The stiffness coefficients used for this first simulation correspond to those of a conventional UM with compliant actuator grasping a rigid object, namely (in N.m ${ }^{-1}$ ) : $k_{p}=0$, $k_{2}=k_{3}=k_{4}=0, k_{1}=1, k_{\rho}=10^{3}$ and $k_{c}=10^{6}$.

The three stages of the grasping process can be clearly seen. When there is no contact, the actual length $\rho$ of the actuator is equal to its commanded value $\rho_{0}$ and the first phalanx (angle $\theta_{p}$ ) is closing while the second one remains in its initial position. Then, when the first contact is established, $\theta_{p}$ remains equal to $\theta_{p c}$ and the second phalanx is closing $\left(\theta_{q}\right.$ increases). Finally, when the object is grasped the configuration is set, the actual length of the actuator becomes different from $\rho_{0}$ and a force equal to $k_{\rho}\left(\rho-\rho_{0}\right)$ is applied on the object. It is important to mention that the different sections of the curves are not lines, the relation between $\rho_{0}$ and $\left(\theta_{p}, \theta_{q}\right)$ being nonlinear.

Figure 5 shows the possible configurations taken by three different mechanisms grasping a rigid object and by a mechanism grasping a compliant object.

a. represents the mechanism used in Fig. 4 grasping a rigid object (its stiffness coefficient is $k_{c}=10^{6} \mathrm{~N} . \mathrm{m}^{-1}$ ). It can be observed that the mechanism is closing or opening one phalanx at a time. When both contacts are established (i.e., when the point $\left(\theta_{p c}, \theta_{q c}\right)$ is reached), the configuration is completely set.

b. still represents the same mechanism, but the grasped object is now compliant, its stiffness being half of that of the actuator $\left(k_{c}=500 \mathrm{~N} \cdot \mathrm{m}^{-1}\right.$ and $\left.k_{\rho}=10^{3} \mathrm{~N} \cdot \mathrm{m}^{-1}\right)$. The curve corresponds to (a) when no contact is established (white area) and when only the first contact is established (pale gray area). However, when the second contact is set, the mechanism can enter in the dark gray 
area, i.e., the mechanism can keep on closing on itself by squeezing the object.

c. corresponds to a compliant mechanism. Each passive joint has a finite stiffness, however the stiffness related to the first phalanx $\left(k_{p}=1 \mathrm{n} \cdot \mathrm{rad}^{-1}\right)$ is lower that the stiffness related to the second phalanx $\left(k_{1}=2 \mathrm{~N} \cdot \mathrm{rad}^{-1}\right.$ and $\left.k_{2,3,4}=1 \mathrm{~N} \cdot \mathrm{rad}^{-1}\right)$. Even if with these values, the behaviour of the compliant mechanism is close to that of the conventional mechanism, it can be observed, that a slight coupling between the motions of the two phalanges appears when no contact is established (The first section of the dashed line is not exactly horizontal).

d. also corresponds to a compliant mechanism. In this case, the design of the gripper is poor because the stiffness related to the first phalanx $\left(k_{p}=4 \mathrm{~N} \cdot \mathrm{rad}^{-1}\right)$ is too high compared to the stiffness related to the second phalanx $\left(k_{1}=1 \mathrm{~N} \cdot \mathrm{rad}^{-1}\right.$ and $\left.k_{2,3,4}=0 \mathrm{~N} \cdot \mathrm{rad}^{-1}\right)$ such that both phalanges are closing simultaneously. With this kind of mechanism, a risk exists that the second phalanx establishes the contact first; and even pokes the object. Graphically in this case, the line composed with crosses would not pass through point $\left(\theta_{p c}, \theta_{q c}\right)$.

\subsubsection{Quasi-static simulation}

Simulation: At each step, the configuration of the mechanism is calculated as a function of the previous configuration and the variation of the commanded value of the actuator $\Delta \rho_{0}$. This is written as $\psi \leftarrow \psi+\mathbf{T} \Delta \rho_{0}$.

The computation of this model is very fast, less than $0.3 \mathrm{~ms}$ in our Matlab program, however the accuracy depends on the magnitude of the increments $\Delta \rho_{0}$. In a context of simulation, these round-off errors are added step after step, but in a control application, since the parameters can be reinitialized using some sensors, the use of the QSM is suitable. Hence, it is important to keep in mind that the dynamic effects are neglected, therefore the variations have to remain small.

Results: The results provided by the QSM are similar to those of the KSM, except for the round-off errors. Figures 6 shows the magnitude of these errors for the computation of $\theta_{p}$ for different magnitudes of $\Delta \rho_{0}\left(\Delta \rho_{0}=0.2 \mathrm{~mm}\right.$ (100 iterations, $30 \mathrm{~ms}), \Delta \rho_{0}=20 \mu \mathrm{m}$ (1000 iterations, $\left.0.20 \mathrm{~s}\right)$, $\Delta \rho_{0}=2 \mu \mathrm{m}\left(10^{4}\right.$ iterations, $\left.2 \mathrm{~s}\right)$ and $\Delta \rho_{0}=0.20 \mu \mathrm{m}\left(10^{5}\right.$ iterations, $20 \mathrm{~s})$.

First, it is interesting to notice that the error in $\theta_{p}$ is growing faster in the first part of the graph corresponding to the non-contact section. Indeed, in this part of the curves, $\theta_{p}$ is varying and therefore the error (that is related to the magnitude of $\Delta \theta_{p}$ ) is larger. Then, when $\theta_{p}$ is set (one and two contacts), $\Delta \theta_{p}$ is close to zero and thus, the growth of the error is smaller.

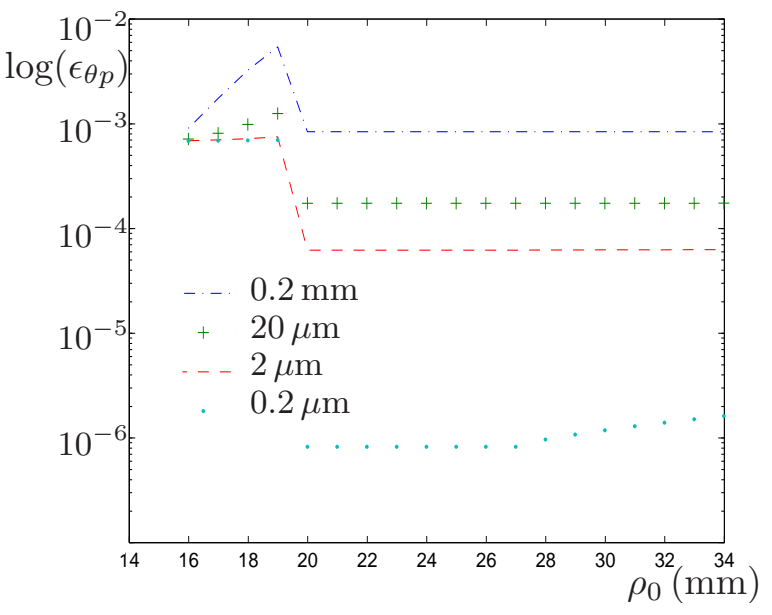

Figure 6. Discrepancy in $\theta_{p}$ between KSM and QSM for different $\Delta \rho_{0}$.

The second and the major source of inaccuracy in the use of the QSM comes from the step where the contact is established since the test has to be performed at the end of the step, so that the object can be squeezed up to $\approx l_{i} \Delta \theta_{i}$; which is another obvious advantage of using small increments. On the other hand, the fact that the contact is established for a certain value of $\theta_{i}$ has the advantage of "reinitializing" the round-off errors at this step.

\subsection{Conclusion about the simulation}

The comparison of the KSM and the QSM has shown their respective advantages and drawbacks. The constant and small computational time of the QSM makes it highly suitable in a context of real-time control of a manipulator. Hence, with the use of some sensors on this manipulator, the problem of the round-off errors disappears. The only remaining problem is the establishment of the contact. However, it is also interesting to notice that the presence of sensors would make the knowledge of the contact points $\boldsymbol{p}$ and $\boldsymbol{q}$ not necessary and therefore, would enable a simpler and more general use of the models. In practice depending on the relative stiffness of the actuator and the object, the finger may penetrate into the latter at a maximal depth of the increment.

The KSM, with kinematic parameters as well as static parameters that enable a detailled modelling of the mechanism, offers very accurate results and can be useful for the analysis and the design of an UM.

\section{Conclusions}

In this paper, the kinematostatic and quasi-static models of compliant mechanisms are applied to underactuated mechanisms. This application brings some interesting contributions to the modelling of compliant mechanisms. First, the theoretical models are extended to the case of actuated dependent 
joints. Second, the case of external loads that are function of the configuration of the mechanism has been studied and has shown that it was equivalent to a reduction of degree of mobility in the system $\{$ mechanism + grasped object\}. On the other hand, since the equivalence between the general formulation of the static equilibrium of a compliant parallel mechanism and the kinetostatic relation of the underaction theory was proven, the former can as well be used as the starting point for the study of the grasp stability, the force isotropy or any other important property of grasping hands.

Then, in the application, a new possibility offered by these models is illustrated: the configuration of an underactuated finger can be described even when it is not in contact with the object to be grasped ${ }^{1}$. All degrees of mobility become function of the single commanded value of the actuator.

Acknowledgements. The authors would like to acknowledge the financial support of the Natural Sciences and Engineering Research Council of Canada (NSERC) as well as the Canada Research Chair (CRC) Program.

Edited by: J. L. Herder

Reviewed by: two anonymous referees

\section{References}

Birglen, L.: An Introduction to the Analysis of Linkage-driven Compliant Underactuated Fingers, in: Proceedings of the ASME Design Engineering Technical Conferences, 2006.

Birglen, L., Laliberté, T., and Gosselin, C. M.: Underactuated Robotic Hands, Spr. Tra. Adv. Robot., 40, 2008.

Boudreault, E. and Gosselin, C. M.: Design of Sub-Centimetre Underactuated Compliant Grippers, in: Proceedings of the ASME Design Engineering Technical Conferences, Philadelphia, PA, USA, 10-13 September 2006.
Carricato, M.: Contributi teorici per l'analisi statica dei meccanismi con elementi elastici, Master's Thesis, Università degli Studi di Bologna, Italy, 1998.

Cho, W., Tesar, D., and Freeman, R. A.: The Dynamic and Stiffness Modeling of General Robotic Manipulator Systems with Antagonistic Actuation, IEEE Int. Conference on Robotics and Automation, 1380-1387, 1989.

Doria, M. and Birglen, L.: Design of an Underactuated Compliant Gripper for Surgery Using Nitinol, Journal of Medical Devices, 3(1), 011007, 2009.

Griffis, M. W. and Duffy, J.: Kinestatic Control: a Novel Theory for Simultaneously Regulating Force and Displacement, J. Mech. Des.-T. ASME, 113(4), 508-515, 1991.

Kragten, G. A., Herder, J. L., and Schwab, A. L.: On the Influence of Contact Geometry on Grasp Stability, in: ASME Design Engineering Technical Conferences and Computer and Information in Engineering Conference, p. 49400, Brooklyn, New York, USA, 3-6 August 2008.

Krut, S.: A Force-Isotropic Underactuated Finger, in: IEEE International Conference on Robotics and Automation, 2325-2330, Barcelona, Spain, 18-22 April 2005.

Lotti, F., Tiezzi, P., Vassura, G., Biagiotti, L., Palli, G., and Melchiorri, C.: Development of UB hand 3: Early results, in: IEEE International Conference on Robotics and Automation, 44884493, Barcelona, Spain, 18-22 April 2005.

Quennouelle, C. and Gosselin, C. M.: Stiffness Matrix of Compliant Parallel Mechanisms, Advances in Robot Kinematics: Analysis and Design, 331-341, 2008.

Quennouelle, C. and Gosselin, C. M.: A Quasi-Static Model for Planar Compliant Parallel Mechanisms, Journal of Mechanisms and Robotics, 1(2), 021012, 2009.

Su, H.-J.: A Pseudorigid-Body 3R Model for Determining Large Deflection of Cantilever Beams Subject to Tip Loads, Journal of Mechanisms and Robotics, 1(2), 021008, 2009.

Sun, L., Liang, C. G., and Liao, Q. Z.: An inverse Static Force Analysis of a Special Planar Three-Spring System, Mech. Mach. Theory, 32(5), 609-615, 1997.

\footnotetext{
${ }^{1}$ Even in a conventional $n$-DOF underactuated mechanism, at least $n-1$ springs have to be added in order to set the configuration of the mechanism when no contact is established.
} 